\title{
Levantamento Fitossociológico em Pastagens ${ }^{1}$
}

\author{
Phyto-Sociological Assessment of Pasture
}

\author{
INOUE, M.H. ${ }^{2}$, SILVA, B.E. ${ }^{3}$, PEREIRA, K.M. ${ }^{3}$, SANTANA, D.C. ${ }^{4}$ CONCIANI, P.A. ${ }^{5}$ e \\ SZTOLTZ, C.L. ${ }^{3}$
}

\begin{abstract}
RESUMO - O objetivo do presente trabalho foi identificar a comunidade daninha presente em áreas de pastagens. O levantamento fitossociológico foi realizado nos meses de novembro e dezembro de 2009 em três regiões: duas localizadas no município de Tangará da Serra-MT (região $\mathrm{A}$ = áreas próximas à cidade; e região $\mathrm{B}=$ áreas presentes no Assentamento Antonio Conselheiro) e uma no município de Barra do Bugres-MT (região C). Em cada região foram analisadas cinco propriedades, tendo cada uma 10 parcelas de $25 \mathrm{~m}^{2}$. Nas parcelas, foram realizadas a contagem e identificação das espécies daninhas. Os dados foram analisados por meio de cálculos de densidade, frequência, abundância, densidade relativa, frequência relativa, abundância relativa, índice de valor de importância (IVI) e índice de similaridade. Foram identificadas 38 espécies daninhas, distribuídas em 18 famílias, sendo Asteraceae (7), Fabaceae (6), Arecaceae (3), Euforbiaceae (3) e Poaceae (3) as mais representativas em número de espécies. As espécies que mais ocorreram na área foram: região A - Sida spp. (IVI 127,93) e Eragrostis plana (IVI: 42,18); região B - Eragrostis plana (IVI: 54,78), Mimosa wedelliana (IVI: 52,39) e Sida spp. (IVI: 50,30); e região C - Sida spp. (IVI: 73,92) e Mimosa wedelliana (IVI: 26,55). Houve similaridade expressiva entre as regiões A e B $(52,63 \%)$ e entre as regiões B e C $(50,98 \%)$.
\end{abstract}

Palavras-chave: plantas daninhas, índice de valor de importância, similaridade, Brachiaria spp.

\begin{abstract}
The phyto-sociological survey was conducted during November and December, 2009 in three regions: two located in Tangará da Serra-MT (region $A=$ areas surrounding the town; and region $B=$ areas in the Antonio Conselheiro Settlement and one area in Barra of the Bugres-MT (region C). Five properties were analyzed in each region, each containing 10 plots of $25 \mathrm{~m}^{2}$. The weed species were counted and identified in the plots. Data were analyzed by calculating density, frequency, abundance, relative density, relative frequency, relative abundance, importance value index (IVI), and similarity index. Thirty-eight weed species were identified, distributed among 18 families, with Asteraceae (7), Fabaceae (6), Arecaceae (3), Euforbiaceae (3) and Poaceae (3) being the most representative in number of species. The species most frequently found were: region A - Sida spp. (IVI: 127.93) and Eragrostis plana (IVI: 42.18); region B - Eragrostis plana (IVI: 54.78), Mimosa wedelliana (IVI: 52.39), and Sida spp. (IVI: 50.30); and region C-Sida spp. (IVI: 73.92), and Mimosa wedelliana (IVI: 26.55). A significant similarity was found between regions $A$ and $B(52.63 \%)$ and between regions $B$ and $C(50.98 \%)$.
\end{abstract}

Keywords: weeds, importance value index, similarity, Brachiaria spp.

1 Recebido para publicação em 27.4.2011 e aprovado em 28.10.2011.

${ }^{2}$ Engo-Agro. ., D.Sc., Dep. de Agronomia, Universidade do Estado de Mato Grosso, Rod. MT 358, Km 07, Caixa Postal 287, 78300000 Tangará da Serra-MT, Brasil, <miriamhinoue@hotmail.com>; ${ }^{3}$ Engo-Agro-., Universidade do Estado do Mato Grosso, Tangará da Serra-MT; ${ }^{4}$ Pós-Graduação em Agricultura, Universidade Estadual Paulista “Júlio de Mesquita Filho”, Botucatu-SP, Brasil; ${ }^{5}$ Graduação em Agronomia, Universidade do Estado do Mato Grosso, Tangará da Serra-MT, Brasil.

Planta Daninha, Viçosa-MG, v. 30, n. 1, p. 55-63, 2012 


\section{INTRODUÇÃO}

O Brasil detém o segundo maior rebanho comercial de bovinos no mundo, com cerca de 205 milhões de cabeças; a maioria da carne produzida no País tem origem nos animais mantidos exclusivamente em pastos (Correio do Brasil, 2010). Assim, a formação de boas pastagens assume real importância, tornandose a melhor opção para a alimentação do rebanho nacional, pois, além de constituir-se no alimento disponível com menor custo, oferece todos os nutrientes necessários para um bom desempenho dos animais (Costa et al., 2008).

A falta de manejo ou o manejo inadequado de pastagens é uma das hipóteses que explicam o processo de degradação que vem sendo observado (Drumond, 2008). Estima-se que $80 \%$ dos 50 a 60 milhões de hectares de pastagens, cultivadas no Brasil Central, encontramse em algum estádio de degradação (Drumond, 2008; Albuquerque et al., 2009).

Um dos problemas consequentes da degradação pelo manejo inadequado das pastagens é a infestação por plantas daninhas, as quais possuem grande capacidade de interferir com as gramíneas cultivadas, uma vez que, devido ao seu comportamento oportunista, ocupam espaços deixados pelas forrageiras (Dias Filho, 1998). A consequência desse processo é a redução da capacidade de lotação das pastagens, o que dificulta a intensificação do uso e a busca de uma pecuária mais produtiva (Lorenzi, 2000).

Por conseguinte, permanece crescente a necessidade do estabelecimento de novas estratégias que promovam a redução da comunidade daninha e mantenha a longevidade produtiva a longo prazo, em níveis aceitáveis de infestação, sendo fundamental para a sustentabilidade da atividade na região e para a lucratividade (Homma et al., 2006). Contudo, a primeira etapa para se definir um programa adequado no manejo de plantas daninhas em pastagens é a identificação e caracterização (Mascarenhas et al., 2009), visando conhecer as características morfofisiológicas, anatômicas, ecológicas e a habilidade competitiva das espécies infestantes (Svicero et al., 2008). Portanto, a partir do levantamento adequado das infestantes, será possivel traçar estratégias eficientes, visando ao manejo dessas espécies.

Particularmente no Estado de Mato Grosso, apesar da grande necessidade do manejo de plantas daninhas em áreas de pastagens, não há dados referentes às principais espécies que compõem essas áreas. Nesse sentido, o objetivo deste trabalho foi identificar as espécies daninhas presentes em pastagens localizadas nos municípios de Tangará da Serra e Barra do Bugres, MT.

\section{MATERIAL E MÉTODOS}

O levantamento foi realizado em pastagens de Brachiaria spp., nos municípios de Tangará da Serra e Barra do Bugres, pertencentes ao Estado de Mato Grosso, entre os meses de novembro e dezembro de 2009. As coletas de dados foram realizadas em 15 propriedades, sendo cinco localizadas em Tangará da Serra, denominada região A, cinco no Assentamento Antonio Conselheiro, denominado região B, que pertence também ao mesmo município, e cinco em Barra do Bugres, denominada região C (Tabela 1).

Para o estudo fitossociológico foi utilizada uma forma adaptada ao método dos quadrados isolados (Braun-Blanquet, 1950), com amostragem de 10 parcelas aleatórias em cada propriedade. A área de cada parcela foi delimitada por uma linha de barbante com uma haste fixada a cada $5 \mathrm{~m}$, constituindo uma área de $25 \mathrm{~m}^{2}$, totalizando $250 \mathrm{~m}^{2} \mathrm{em}$ cada propriedade. Em cada parcela amostrada, as plantas foram identificadas segundo a família, o gênero e a espécie.

A análise da flora daninha foi feita por meio de cálculos de frequência, que expressa a intensidade de ocorrência de uma espécie pelas áreas, em porcentagem; densidade, que se refere ao número de plantas por unidade de área em cada espécie, expressa em plantas $\mathrm{m}^{-2}$; e abundância, que informa sobre as espécies cujas plantas ocorrem concentradas em determinados pontos. Além desses cálculos, foi realizada a análise de frequência relativa, densidade relativa e abundância relativa, que resultaram no valor da importância. O Îndice de Valor de Importância (IVI) estabelece um parâmetro de integração das variáveis parciais, de forma a combiná-los em 
Tabela 1 - Localização das áreas de pastagens analisadas no levantamento fitossociológico

\begin{tabular}{|c|c|c|c|c|c|}
\hline \multirow{2}{*}{ Região } & \multirow{2}{*}{ Propriedade } & \multicolumn{2}{|c|}{ Coordenada } & \multirow{2}{*}{ Pastagem } & \multirow{2}{*}{ Manejo* } \\
\hline & & Latitude & Longitude & & \\
\hline \multirow{5}{*}{ A } & Tuiutí & S $14^{\circ} 39^{\prime} 47,8^{\prime \prime}$ & O $57^{\circ} 22^{\prime} 14,8^{\prime \prime}$ & B. brizantha; $B$. humidicola & $\underline{1} /$ \\
\hline & WD & S $14^{\circ} 40^{\prime} 10,3^{\prime \prime}$ & O $57^{\circ} 22^{\prime} 14,8^{\prime \prime}$ & B. brizantha; $B$. humidicola & $\underline{2} /$ \\
\hline & Sítio Souza & S $14^{\circ} 33^{\prime} 48,2^{\prime \prime}$ & O $57^{\circ} 27^{\prime} 33,8^{\prime \prime}$ & B. brizantha & $1 /$ \\
\hline & N. Sra. Aparecida & S $14^{\circ} 15^{\prime} 17,5^{\prime \prime}$ & O $57^{\circ} 28^{\prime} 13,3^{\prime \prime}$ & B. humidicola & $\underline{1 /}$ \\
\hline & São José & $\mathrm{S} 14^{\circ} 35^{\prime} 05,1^{\prime \prime}$ & O 57²28'16,2" & Brachiaria sp. & $\underline{1 /}$ \\
\hline \multirow{5}{*}{ B } & Bela Vista & S $14^{\circ} 40^{\prime} 00,5^{\prime \prime}$ & O 57044'41,6" & B. brizantha & $\underline{3} /$ \\
\hline & São José & S $14^{\circ} 41^{\prime} 09,8^{\prime \prime}$ & O $57^{\circ} 45^{\prime} 05,4^{\prime \prime}$ & B. brizantha & $\underline{3} /$ \\
\hline & Chaparal & $\mathrm{S} 14^{\circ} 38^{\prime} 49,1^{\prime \prime}$ & O 57044'01,1" & B. brizantha & $\underline{3} /$ \\
\hline & Boa Esperança & S $14^{\circ} 38^{\prime} 45,3^{\prime \prime}$ & O $57^{\circ} 44^{\prime} 09,9^{\prime \prime}$ & B. decumbens & $\underline{3} /$ \\
\hline & Santa Amália & S 14³6'51,1" & O $57^{\circ} 41^{\prime} 50,6^{\prime \prime}$ & Brachiaria sp. & $\underline{2} /$ \\
\hline \multirow{5}{*}{$\mathrm{C}$} & Paraná & $\mathrm{S} 15^{\circ} 06^{\prime} 06,1^{\prime \prime}$ & O $57^{\circ} 17^{\prime} 17,5^{\prime \prime}$ & B. decumbens & $\underline{2} /$ \\
\hline & Barreiro rico & S $15^{\circ} 03^{\prime} 56,4^{\prime \prime}$ & O $57^{\circ} 14^{\prime} 08,2^{\prime \prime}$ & B. brizantha; $B$. humidicola & $\underline{2} /$ \\
\hline & N. Sra. Aparecida & S $15^{\circ} 07^{\prime} 22,7^{\prime \prime}$ & O $57^{\circ} 17^{\prime} 34,3^{\prime \prime}$ & B. brizantha & $\underline{1} /$ \\
\hline & Rondônia & S 145' $17,2^{\prime \prime}$ & O $57^{\circ} 10^{\prime} 38,3^{\prime \prime}$ & B. decumbens & $\underline{2} /$ \\
\hline & Santa Terezinha & 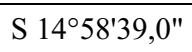 & O $57^{\circ} 11^{\prime} 31,0^{\prime \prime}$ & Brachiaria sp. & $\underline{2} /$ \\
\hline
\end{tabular}

${ }^{1 /}$ Utilização de dois ou mais métodos de manejo de plantas daninhas na área (capina manual, roçada e/ou herbicidas). ${ }^{2 /}$ Utilização de um método de manejo de plantas daninhas na área (capina manual, roçada ou herbicidas). ${ }^{3 /}$ Sem utilização de métodos de manejo de plantas daninhas.

expressão única e simples, expondo a importância relativa de cada espécie melhor que qualquer outro parâmetro fitossociológico (Lamprecht, 1964).
No cálculo desses parâmetros foram utilizadas as seguintes fórmulas (Brandão et al., 1998):

$$
\text { Frequência }=\frac{\mathrm{n}^{\mathrm{o}} \text { de parcelasque contêma espécie } \mathrm{x} 100}{\mathrm{n}^{\mathrm{o}} \text { total de parcelas utilizadas }}
$$

Densidade $=\frac{\mathrm{n}^{\mathrm{o}} \text { total de indivíduos por espécie }}{\text { área total da coleta }}$

$$
\text { Abundância }=\frac{\mathrm{n}^{\circ} \text { total de indivíduos por espécie }}{\mathrm{n}^{\mathrm{o}} \text { total de parcelas contendo a espécie }}
$$

Frequência relativa (F.r.) $=\frac{\text { frequência da espécie } x 100}{\text { frequência total de todas as espécies }}$

$$
\text { Densidade relativa (D.r.) }=\frac{\text { densidade da espécie } \mathrm{x} 100}{\text { densidade total das espécies }}
$$


Abundância relativa (A.r.) $=\frac{\text { abundância x } 100}{\text { abundância total de todas as espécies }}$

IVI = F.r. + D.r. + A.r.

Para analisar a similaridade das populações de espécies daninhas das áreas de pastagens, entre as regiões A, B e C, foi utilizado o Índice de Similaridade (IS) (Sorensen, 1972). Para o seu cálculo, utilizou-se a seguinte fórmula:

$$
I S=(2 a / b+c) \times 100
$$

em que $a$ é o número de espécies comuns às duas áreas; e $b, c=$ número total de espécies nas duas áreas comparadas. O IS é expresso em porcentagem, sendo máximo (100\%) quando todas as espécies são comuns às duas áreas e mínimo $(0 \%)$ quando não há espécies comuns.

\section{RESULTADOS E DISCUSSÃO}

Foram identificadas 38 espécies de plantas daninhas, pertencentes a 18 famílias botânicas (Tabela 2). As famílias mais representativas do levantamento fitossociológico, no que se refere a número de espécies, foram Asteraceae, Fabaceae e Arecaceae, com maior ocorrência na região B (Tabela 3 ).

$\mathrm{Na}$ família Asteraceae foram encontradas as espécies Vernonia brasiliana, Galinsoga sp., Tridax procumbens, Pluchea sagittalis, Emilia sonchifolia, Xanthium strumarium e Dasyphyllum brasiliense (Tabela 2). Na família Fabaceae estão incluídas as espécies Bauhinia forficata, Mimosa pudica, Mimosa wedelliana, Senna obtusiofolia, Senna occidentalis e Acacia plumosa (Tabela 2). Em seguida veio a família Arecaceae, com as espécies Astrocaryum vulgare, Attalea speciosa e Attalea phalerata (Tabela 2).

Tuffi Santos et al. (2004) destacam a importância da espécie $M$. pudica, que se caracteriza por apresentar grande quantidade de espinhos em seus ramos, o que impede o pastejo dos animais nas suas proximidades ou pode causar ferimentos nestes. Do mesmo modo, Senna occcidentalis, popularmente

Tabela 2 - Espécies de plantas daninhas encontradas no levantamento fitossociológico em pastagens, organizadas por família, nome científico e nome popular

\begin{tabular}{|c|c|c|}
\hline Família & Nome científico & Nome vulgar \\
\hline \multirow{2}{*}{ Amaranthaceae } & Alternanthera tenella & Apaga-fogo \\
\hline & Amaranthus spinosus & Caruru-de-espinho \\
\hline Apocyanaceae & Aspidosperma sp. & Peroba \\
\hline \multirow{3}{*}{ Arecaceae } & Astrocaryum vulgare & Tucum-bravo \\
\hline & Attalea speciosa & Babaçu \\
\hline & Attalea phalerata & Bacuri \\
\hline \multirow{7}{*}{ Asteraceae } & Dasyphyllum brasiliense & Espinho-agulha \\
\hline & Emilia sonchifolia & Falsa-serralha \\
\hline & Galinsoga sp. & Picão-branco \\
\hline & Pluchea sagittalis & Quitoco \\
\hline & Tridax procumbens & Erva-de-touro \\
\hline & Vernonia brasiliana & Assa-peixe \\
\hline & Xanthium strumarium & Carrapicho-bravo \\
\hline Bignoniaceae & Amphilophium paniculatum & Cipó-quatro-quina \\
\hline Boraginaceae & Heliotropium indicum & Crista-de-galo \\
\hline Bromeliaceae & Ananas ananassoides & Abacaxi-do-cerrado \\
\hline Chenopodiaceae & Chenopodium ambrosiodies & Erva-de-santa-maria \\
\hline \multirow{2}{*}{ Convolvulaceae } & Ipomoea grandifolia & Corda-de-viola \\
\hline & Ipomoea quamoclit & Flor-de-cardeal \\
\hline Cucurbitaceae & Cucumis anguria & Maxixe \\
\hline \multirow{2}{*}{ Cyperaceae } & Cyperus escunlentus & Tiririca \\
\hline & Rhynchospora aurea & Capim-navalha \\
\hline \multirow{3}{*}{ Euforbiaceae } & Cnidoscolus cnicodendron & Cansanção \\
\hline & Chamaessyce hirta & Erva-de-santa-luzia \\
\hline & Phyllanthus tenellus & Quebra-pedra \\
\hline \multirow{6}{*}{ Fabaceae } & Acacia plumosa & Arranha-gato \\
\hline & Bauhinia sp. & Pata-de-vaca \\
\hline & Mimosa pudica & Malícia \\
\hline & Mimosa wedelliana & Dorme-dorme \\
\hline & Senna obtusiofolia & Fedegoso \\
\hline & Senna occidentalis & Fedegoso \\
\hline Malvaceae & Sida spp. & Guanxuma \\
\hline Monimiaceae & Siparuna apiosyce & Limão-bravo \\
\hline Papilionoideae & Crotalaria incana & Guizo-de-cascavel \\
\hline \multirow{3}{*}{ Poaceae } & Andropogon bicornis & Rabo-de-burro \\
\hline & Digitaria insularis & Amargoso \\
\hline & Eragrostis plana & Capim-annoni 2 \\
\hline Tiliaceae & Triumfetta bartramia & Carrapichão \\
\hline
\end{tabular}

Planta Daninha, Viçosa-MG, v. 30, n. 1, p. 55-63, 2012 
conhecida como fedegoso, é uma planta considerada tóxica para bovinos e outros animais herbivoros. Os principais sintomas de intoxicação em bovinos são diarreia, fraqueza, tremor e desequilíbrio (Pott \& Pott, 1994).

Tabela 3 - Número total de espécies de plantas daninhas, organizadas por família, encontradas em pastagens nas regiões $\mathrm{A}, \mathrm{B}$ e C

\begin{tabular}{|c|c|c|c|c|}
\hline \multirow{2}{*}{ Família } & \multicolumn{3}{|c|}{$\begin{array}{c}\text { Número de espécies de plantas } \\
\text { daninhas }\end{array}$} & \multirow{2}{*}{ Total } \\
\hline & Região A & Região B & Região C & \\
\hline Asteraceae & 3 & 5 & 2 & 7 \\
\hline Fabaceae & 2 & 5 & 4 & 6 \\
\hline Arecaceae & 1 & 3 & 2 & 3 \\
\hline Euforbiaceae & 2 & 3 & 1 & 3 \\
\hline Poaceae & 1 & 2 & 2 & 3 \\
\hline Convolvulaceae & 0 & 2 & 1 & 2 \\
\hline Amaranthaceae & 0 & 0 & 2 & 2 \\
\hline Cyperaceae & 0 & 0 & 2 & 2 \\
\hline Bignoniaceae & 1 & 1 & 1 & 1 \\
\hline Malvaceae & 1 & 1 & 1 & 1 \\
\hline Apocyanaceae & 0 & 1 & 1 & 1 \\
\hline Monimiaceae & 0 & 1 & 1 & 1 \\
\hline Boraginaceae & 0 & 0 & 1 & 1 \\
\hline Bromeliaceae & 0 & 1 & 0 & 1 \\
\hline Chenopodiaceae & 0 & 0 & 1 & 1 \\
\hline Cucurbitaceae & 0 & 0 & 1 & 1 \\
\hline Papilionoideae & 0 & 1 & 0 & 1 \\
\hline Tiliaceae & 0 & 1 & 0 & 1 \\
\hline Total & 11 & 27 & 23 & 38 \\
\hline
\end{tabular}

Autores como Carvalho \& Pitelli (1992) encontraram resultados semelhantes no levantamento fitossociológico realizado em áreas de pastagens no município de Selvíria, no Estado de Mato Grosso do Sul, onde as famílias Fabaceae e Asteraceae apresentaram o maior número de espécies nas áreas estudadas. Levantamentos realizados por Lara et al. (2003) em áreas de pastagens de várzeas no Estado de Minas Gerais, às margens do rio São Francisco, e por Maciel et al. (2008), ao levantarem aspectos florísticos em dois gramados de Paspalum notatum, no município de Assis - SP, constataram a presença na família Asteraceae de maior número de espécies. No entanto, em outros levantamentos, como o de Tuffi Santos et al. (2004), realizado em áreas de pastagens degradadas sob condições de várzea, no município de Leopoldina-MG, houve maior presença da família Poaceae seguida de Asteraceae. Similarmente, no levantamento fitossociológico em pastagens de várzea do município de Autazes-AM, Galvão et al. (2011) verificaram que as famílias mais importantes em número de espécies foram Poaceae e Cyperaceae. Trabalhos de Silva et al. (2008) realizados no parque da Criança, localizado no centro de Campina Grande (PB), citam 67 táxons específicos, distribuídos em 18 famílias de espécies daninhas; as famílias Asteraceae e Fabaceae ficaram, respectivamente, com o segundo e quarto maiores números de espécies.

A região $B$ apresentou a maior biodiversidade, com o total de 27 espécies daninhas, seguida pelas regiões $\mathrm{C}$ e A, respectivamente, com 21 e 11 espécies (Tabela 3). Essa constatação deve-se provavelmente aos reflexos dos diferentes sistemas de manejo e práticas culturais, aplicados pelos agricultores em cada região, pois essas variáveis influenciam a dinâmica da comunidade daninha (Oryokot et al., 1997). Nesse contexto, a região B apresenta cultivo caracterizado como rudimentar, e a região A, cultivo tecnificado, o que justifica a alta biodiversidade de espécies daninhas encontradas na primeira (Tabela 1).

Constatou-se, na região A, que a espécie de maior importância foi Sida spp., com IVI igual a 127,93 (Tabela 4), estando presente em todas as parcelas, com frequência de $100 \%$ e densidade de 1,57 planta $\mathrm{m}^{-2}$ (Tabela 4). Esse fato evidencia a necessidade de estratégias de manejo dessa espécie em áreas de pastagens na região. A espécie Eragrostis plana foi a segunda de maior importância, com IVI igual a 42,18 (Tabela 4) e valor de abundância superior ao de Sida spp., de 40,13 (Tabela 4), o que caracteriza a ocorrência concentrada da espécie em determinados pontos, revelando o aparecimento dela em reboleiras. No entanto, as espécies Galinsoga sp., Mimosa wedelliana e Vernonia brasiliana apresentaram IVI expressivos: 27,66, 25,64 e 24,90, respectivamente 
Tabela 4 - Relação das espécies de plantas daninhas encontradas em pastagens na região A

\begin{tabular}{|c|c|c|c|c|c|c|c|c|c|}
\hline \multirow{2}{*}{ Espécie } & № & $\mathrm{N}^{\mathrm{o}}$ & \multirow{2}{*}{ Fre. } & \multirow{2}{*}{ Den. } & \multirow{2}{*}{ Abu. } & \multirow{2}{*}{ Frr. } & \multirow{2}{*}{ Der. } & \multirow{2}{*}{ Abr. } & \multirow{2}{*}{ IVI } \\
\hline & ind. & par. & & & & & & & \\
\hline Sida spp. & 1.960 & 50 & 100 & 1,57 & 39,20 & 39,37 & 63,70 & 24,86 & 127,93 \\
\hline Eragrostis plana & 321 & 8 & 16 & 0,26 & 40,13 & 6,30 & 10,43 & 25,45 & 42,18 \\
\hline Galinsoga sp. & 212 & 15 & 30 & 0,17 & 14,13 & 11,81 & 6,89 & 8,96 & 27,66 \\
\hline Mimosa wedelliana & 173 & 8 & 16 & 0,14 & 21,63 & 6,30 & 5,62 & 13,71 & 25,64 \\
\hline Vernonia brasiliana & 181 & 12 & 24 & 0,14 & 15,08 & 9,45 & 5,88 & 9,57 & 24,90 \\
\hline Cnidoscolus cnicodendron & 106 & 16 & 32 & 0,08 & 6,63 & 12,60 & 3,44 & 4,20 & 20,24 \\
\hline Pluchea sagittalis & 103 & 10 & 20 & 0,08 & 10,30 & 7,87 & 3,35 & 6,53 & 17,75 \\
\hline Bauhinia forficata & 13 & 5 & 10 & 0,01 & 2,60 & 3,94 & 0,42 & 1,65 & 6,01 \\
\hline Phyllanthus tenellus & 5 & 1 & 2 & $0,0^{*}$ & 5,00 & 0,79 & 0,16 & 3,17 & 4,12 \\
\hline Amphilophium paniculatum & 2 & 1 & 2 & $0,0^{*}$ & 2,00 & 0,79 & 0,06 & 1,27 & 2,12 \\
\hline Tridax procumbens & 1 & 1 & 2 & $0,0^{*}$ & 1,00 & 0,79 & 0,03 & 0,63 & 1,45 \\
\hline Total & 3.077 & 127 & 254 & 2,46 & 157,69 & 100,00 & 100,00 & 100,00 & 300,00 \\
\hline
\end{tabular}

№ ind. = número de indivíduos; № par. = número de parcelas; Fre = frequência; Den = densidade; Abu = abundância; Frr. = frequência relativa; Der. = densidade relativa; Abr. = abundância relativa; IVI = índice de valor de importância; $(*)=$ menor que $0,01$.

(Tabela 4). Galinsoga sp. juntamente com Cnidoscolus cnicodendron foram as espécies que apresentaram as maiores frequências após Sida spp., com valores de 30 e 32\%, respectivamente (Tabela 4).

Na região B, a espécie Eragrostis plana foi a de maior abundância, com valor igual a 86,67 (Tabela 5). Sida spp. foi a que ocorreu com maior intensidade (frequência de 64\%), seguida por Attalea speciosa (36\%) e Mimosa wedelliana (34\%) (Tabela 5). Esta última espécie, além de apresentar alta frequência, ocorreu com a maior densidade, ou seja, 0,42 planta $\mathrm{m}^{-2}$ (Tabela 5). Os dados da Tabela 5 indicam ainda que as espécies Eragrostis plana, Mimosa wedelliana e Sida spp. se destacaram, com IVI de 54,78, 52,39 e 50,30, respectivamente, sendo as plantas daninhas mais predominantes na região B. Esse fato deve-se, provavelmente, à carência de manejo de plantas daninhas nessas áreas, o que favoreceu a rápida disseminação dessas espécies.

Constatou-se, na região $\mathrm{C}$, que algumas espécies se destacaram em variáveis isoladas (Tabela 6). Vernonia brasiliana e Xanthium strumarium foram as mais frequentes na área, com níveis de 46\%, seguidas de Mimosa wedelliana, com 32\% (Tabela 6). As espécies
Amaranthus spinosus, Rhynchospora aurea e Cyperus escunlentus apresentaram altos indices de abundância, com 54,5, 43,33 e 42,75 , respectivamente (Tabela 6), indicando serem espécies ocorrentes em reboleiras. Sida spp. apresentou $42 \%$ de frequência, com os maiores valores de densidade $\left(1,15\right.$ planta $\left.\mathrm{m}^{-2}\right)$ e abundância $(68,19)$, em relação às demais espécies, resultando no maior IVI $(73,92)$ (Tabela 6), sendo a espécie mais infestante na área. Comparativamente às regiões $\mathrm{A}$ e $\mathrm{C}$, áreas presentes na região $\mathrm{C}$ apresentam nível intermediário de tecnologia.

Por meio do Índice de Similaridade (IS), foi possivel deduzir as espécies daninhas similares existentes entre as regiões A, B e C. Os maiores IS foram entre as regiões A e B, com 52,63\% (Tabela 7), demonstrando alta homogeneidade entre as duas regiões. Segundo Felfili \& Venturoli (2000), o IS pode ser considerado elevado quando for superior a $50 \%$. Essa similaridade pode ser explicada pela proximidade entre as regiões analisadas, visto que as regiões A e B estão localizadas no mesmo município. É importante ressaltar que 10 das 11 espécies encontradas na região $A$ também foram observadas na região B. Simultaneamente, as regiões B e C apresentaram IS de 50,98\% (Tabela 7), 
Tabela 5 - Relação das espécies de plantas daninhas encontradas em pastagens na região B

\begin{tabular}{|c|c|c|c|c|c|c|c|c|c|}
\hline \multirow{2}{*}{ Espécie } & № & № & \multirow{2}{*}{ Fre. } & \multirow{2}{*}{ Den. } & \multirow{2}{*}{ Abu. } & \multirow{2}{*}{ Frr. } & \multirow{2}{*}{ Der. } & \multirow{2}{*}{ Abr. } & \multirow{2}{*}{ IVI } \\
\hline & ind. & par. & & & & & & & \\
\hline Eragrostis plana & 260 & 3 & 6 & 0,21 & 86,67 & 1,70 & 14,33 & 38,75 & 54,78 \\
\hline Mimosa wedelliana & 525 & 17 & 34 & 0,42 & 30,88 & 9,66 & 28,93 & 13,81 & 52,39 \\
\hline Sida spp. & 465 & 32 & 64 & 0,37 & 14,53 & 18,18 & 25,62 & 6,50 & 50,30 \\
\hline Vernonia brasiliana & 184 & 15 & 30 & 0,15 & 12,27 & 8,52 & 10,14 & 5,48 & 24,15 \\
\hline Attalea speciosa & 93 & 18 & 36 & 0,07 & 5,17 & 10,23 & 5,12 & 2,31 & 17,66 \\
\hline Bauhinia forficata & 54 & 16 & 32 & 0,04 & 3,38 & 9,09 & 2,98 & 1,51 & 13,58 \\
\hline Senna obtusiofolia & 37 & 9 & 18 & 0,03 & 4,11 & 5,11 & 2,04 & 1,84 & 8,99 \\
\hline Tridax procumbens & 28 & 2 & 4 & 0,02 & 14,00 & 1,14 & 1,54 & 6,26 & 8,94 \\
\hline Attalea phalerata & 25 & 10 & 20 & 0,02 & 2,50 & 5,68 & 1,38 & 1,12 & 8,18 \\
\hline Ipomoea grandifolia & 19 & 11 & 22 & 0,02 & 1,73 & 6,25 & 1,05 & 0,77 & 8,07 \\
\hline Dasyphyllum brasiliense & 30 & 4 & 8 & 0,02 & 7,50 & 2,27 & 1,65 & 3,35 & 7,28 \\
\hline Astrocaryum vulgare & 17 & 7 & 14 & 0,01 & 2,43 & 3,98 & 0,94 & 1,09 & 6,00 \\
\hline Cnidoscolus cnicodendron & 12 & 8 & 16 & 0,01 & 1,50 & 4,55 & 0,66 & 0,67 & 5,88 \\
\hline Chamaessyce hirta & 10 & 1 & 2 & 0,01 & 10,00 & 0,57 & 0,55 & 4,47 & 5,59 \\
\hline Pluchea sagittalis & 15 & 2 & 4 & 0,01 & 7,50 & 1,14 & 0,83 & 3,35 & 5,32 \\
\hline Triumfetta bartramia & 10 & 4 & 8 & 0,01 & 2,50 & 2,27 & 0,55 & 1,12 & 3,94 \\
\hline Siparuna apiosyce & 9 & 3 & 6 & 0,01 & 3,00 & 1,70 & 0,50 & 1,34 & 3,54 \\
\hline Ananas ananassoides & 6 & 3 & 6 & $0,0^{*}$ & 2,00 & 1,70 & 0,33 & 0,89 & 2,93 \\
\hline Mimosa pudica & 6 & 2 & 4 & $0,0^{*}$ & 3,00 & 1,14 & 0,33 & 1,34 & 2,81 \\
\hline Andropogon bicornis & 2 & 2 & 4 & $0,0^{*}$ & 1,00 & 1,14 & 0,11 & 0,45 & 1,69 \\
\hline Aspidosperma sp. & 2 & 1 & 2 & $0,0^{*}$ & 2,00 & 0,57 & 0,11 & 0,89 & 1,57 \\
\hline Phyllanthus tenellus & 1 & 1 & 2 & $0,0^{*}$ & 1,00 & 0,57 & 0,06 & 0,45 & 1,07 \\
\hline Ipomoea quamoclit & 1 & 1 & 2 & $0,0^{*}$ & 1,00 & 0,57 & 0,06 & 0,45 & 1,07 \\
\hline Emilia sonchifolia & 1 & 1 & 2 & $0,0^{*}$ & 1,00 & 0,57 & 0,06 & 0,45 & 1,07 \\
\hline Crotalaria incana & 1 & 1 & 2 & $0,0^{*}$ & 1,00 & 0,57 & 0,06 & 0,45 & 1,07 \\
\hline Amphilophium paniculatum & 1 & 1 & 2 & $0,0^{*}$ & 1,00 & 0,57 & 0,06 & 0,45 & 1,07 \\
\hline Senna occidentalis & 1 & 1 & 2 & $0,0^{*}$ & 1,00 & 0,57 & 0,06 & 0,45 & 1,07 \\
\hline Total & 1.815 & 176 & 352 & 1,45 & 223,66 & 100,00 & 100,00 & 100,00 & 300,00 \\
\hline
\end{tabular}

№ ind. = número de indivíduos; № par. = número de parcelas; Fre = frequência; Den = densidade; Abu = abundância; Frr. = frequência relativa; Der. = densidade relativa; Abr. = abundância relativa; IVI $=$ índice de valor de importância. $(*)=$ menor que 0,01 .

demonstrando alta homogeneidade; 13 das 24 espécies da região $C$ estão presentes na região B. Esse resultado pode estar relacionado com a semelhança entre os sistemas de manejo e práticas culturais, utilizados nas duas regiões. Entretanto, as regiões A e C apresentaram IS de 29,41\% (Tabela 7), baixa homogeneidade, que pode ser justificada pelo diferencial de biodiversidade e pela distância entre as duas regiões, ou, em parte, pela ação antrópica. Evidenciou-se ainda que na maioria das áreas da região A foi adotado algum método de manejo de plantas daninhas nos últimos três anos.

Portanto, as famílias Asteraceae e Fabaceae foram as que apresentaram os maiores números de espécies. A espécie Sida spp. foi a que apresentou a maior importância nas regiões $\mathrm{A}$ e $\mathrm{C}$ e a terceira maior na região $\mathrm{B}$. 
Tabela 6 - Relação das espécies de plantas daninhas encontradas em pastagens na região C

\begin{tabular}{|c|c|c|c|c|c|c|c|c|c|}
\hline \multirow{2}{*}{ Espécie } & $\mathrm{N}^{\mathrm{o}}$ & $\mathrm{N}^{\mathrm{o}}$ & \multirow{2}{*}{ Fre. } & \multirow{2}{*}{ Den. } & \multirow{2}{*}{ Abu. } & \multirow{2}{*}{ Frr. } & \multirow{2}{*}{ Der. } & \multirow{2}{*}{ Abr. } & \multirow{2}{*}{ IVI } \\
\hline & ind. & par. & & & & & & & \\
\hline Sida spp. & 1.432 & 21 & 42 & 1,15 & 68,19 & 11,93 & 43,54 & 18,45 & 73,92 \\
\hline Mimosa wedelliana & 369 & 16 & 32 & 0,30 & 23,06 & 9,09 & 11,22 & 6,24 & 26,55 \\
\hline Rhynchospora aurea & 260 & 6 & 12 & 0,21 & 43,33 & 3,41 & 7,91 & 11,73 & 23,04 \\
\hline Xanthium strumarium & 171 & 23 & 46 & 0,14 & 7,43 & 13,07 & 5,20 & 2,01 & 20,28 \\
\hline Vernonia brasiliana & 170 & 23 & 46 & 0,14 & 7,39 & 13,07 & 5,17 & 2,00 & 20,24 \\
\hline Amaranthus spinosus & 109 & 2 & 4 & 0,09 & 54,50 & 1,14 & 3,31 & 14,75 & 19,20 \\
\hline Cyperus escunlentus & 171 & 4 & 8 & 0,14 & 42,75 & 2,27 & 5,20 & 11,57 & 19,04 \\
\hline Senna obtusifolia & 110 & 14 & 28 & 0,09 & 7,86 & 7,95 & 3,34 & 2,13 & 13,43 \\
\hline Acacia plumosa & 121 & 6 & 12 & 0,10 & 20,17 & 3,41 & 3,68 & 5,46 & 12,55 \\
\hline Cnidoscolus cnicodendron & 88 & 13 & 26 & 0,07 & 6,77 & 7,39 & 2,68 & 1,83 & 11,89 \\
\hline Digitaria insularis & 83 & 5 & 10 & 0,07 & 16,60 & 2,84 & 2,52 & 4,49 & 9,86 \\
\hline Senna occidentalis & 56 & 8 & 16 & 0,04 & 7,00 & 4,55 & 1,70 & 1,89 & 8,14 \\
\hline Heliotropium indicum & 39 & 2 & 4 & 0,03 & 19,50 & 1,14 & 1,19 & 5,28 & 7,60 \\
\hline Ipomoea grandifolia & 13 & 9 & 18 & 0,01 & 1,44 & 5,11 & 0,40 & 0,39 & 5,90 \\
\hline Astrocaryum vulgare & 26 & 7 & 14 & 0,02 & 3,71 & 3,98 & 0,79 & 1,01 & 5,77 \\
\hline Siparuna apiosyce & 25 & 2 & 4 & 0,02 & 12,50 & 1,14 & 0,76 & 3,38 & 5,28 \\
\hline Alternanthera tenella & 14 & 2 & 4 & 0,01 & 7,00 & 1,14 & 0,43 & 1,89 & 3,46 \\
\hline Andropogon bicornis & 8 & 1 & 2 & 0,01 & 8,00 & 0,57 & 0,24 & 2,16 & 2,98 \\
\hline Attalea speciosa & 11 & 2 & 4 & 0,01 & 5,50 & 1,14 & 0,33 & 1,49 & 2,96 \\
\hline Amphilophium paniculatum & 4 & 4 & 8 & $0,0^{*}$ & 1,00 & 2,27 & 0,12 & 0,27 & 2,66 \\
\hline Aspidosperma sp. & 5 & 2 & 4 & $0,0^{*}$ & 2,50 & 1,14 & 0,15 & 0,68 & 1,96 \\
\hline Chenopodium ambrosiodies & 1 & 3 & 6 & $0,0^{*}$ & 0,33 & 1,70 & 0,03 & 0,09 & 1,83 \\
\hline Cucumis anguria & 3 & 1 & 2 & $0,0^{*}$ & 3,00 & 0,57 & 0,09 & 0,81 & 1,47 \\
\hline Total & 3.289 & 176 & 352 & 2,63 & 370,55 & 100,00 & 100,00 & 100,00 & 300,00 \\
\hline
\end{tabular}

№ ind. = número de indivíduos; № par. = número de parcelas; Fre = frequência; Den = densidade; Abu = abundância; Frr. = frequência relativa; Der. = densidade relativa; Abr. = abundância relativa; IVI $=$ índice de valor de importância. $(*)=$ menor que 0,01 .

Tabela 7 - Índice de similaridade da composição de plantas daninhas em pastagens, entre as regiões estudadas

\begin{tabular}{|c|c|c|}
\hline Região & A & C \\
\hline C & $29,41 \%$ & - \\
\hline B & $52,63 \%$ & $50,98 \%$ \\
\hline
\end{tabular}

Outras espécies se apresentaram importantes, como Eragrostis plana, nas regiões A e B, Mimosa wedelliana, na região $\mathrm{B}$, e Vernonia brasiliana e Xanthium strumarium, na área $\mathrm{C}$. As regiões A e B, e B e C apresentaram elevada similaridade das espécies de plantas daninhas. Dessa forma, visando ao manejo destas plantas nas regiões levantadas, evidencia-se a importância em conhecer a biologia e direcionar o controle para as principais espécies relatadas neste estudo.

\section{LITERATURA CITADA}

ALBURQUERQUE, C. J. B. et al. Arranjo de plantas para o sorgo forrageiro no semi-árido de Minas Gerais. Belo Horizonte: EPAMIG/CTNM, 2009. (EPAMIG/CTNM, Circular, 58).

BRANDÃO, M.; BRANDÃO, H.; LACA-BUENDIA, J. P. A mata ciliar do rio Sapucaí, município de Santa Rita do Sapucaí-MG: fitossociologia. Daphne, v. 8, n. 4, p. 36-48, 1998.

BRAUN-BLANQUET, J. Sociología vegetal: estudios de las comunidades vegetales. Buenos Aires: Acme Agency, 1950. $444 \mathrm{p}$.

CARVALHO, S. L.; PITELLI, R. A. Levantamento e análise fitossociológica das principais espécies de plantas daninhas de pastagens da região de Selvíria (MS). Planta Daninha, v. 10, n. 1/2, p. 25-32, 1992. 
CORREIO DO BRASIL. Rebanho bovino brasileiro é o segundo maior do mundo. 24, nov. 2010. Disponível em: $<$ http://correiodobrasil.com.br/rebanho-bovino-brasileiro-e-osegundo-maior-do-mundo/192661.> . Acesso em: 3 maio 2011.

COSTA, C. et al. Evolução das pastagens cultivadas e do efetivo bovino no Brasil. Arq. Bras. Med. Veter. Zoot., v. 15, n. 1, p. 8-17, 2008.

DIAS FILHO, M. B. Pastagens cultivadas na Amazônia oriental brasileira: processos e causas de degradação e estratégias de recuperação. In: DIAS, L. E.; MELLO, J. W. V. (Eds). Recuperação de áreas degradadas. Viçosa, MG: Universidade Federal de Viçosa/Sociedade Brasileira de Recuperação de Áreas Degradadas, 1998. p. 135-149.

DRUMOND, L. C. D. Irrigação de pastagens. In: SIMPÓSIO INTERNACIONAL DE PRODUÇÃO DE GADO DE CORTE, 6., 2008, Viçosa. Anais... Viçosa, MG: Universidade Federal de Viçosa, 2008. p. 307-320.

FELFILI, J. M.; VENTUROLI, F. Tópicos em análise de vegetação. Comum. Técn. Flor., v. 2, n. 2, p. 1-34, 2000.

GALVÃO, A. K. L. et al. Levantamento fitossociológico em pastagens de várzea no Estado do Amazonas.

Planta Daninha, v. 29, n. 1, p. 69-75, 2011.

HOMMA, A. K. O. et al. Criação de bovinos de corte no Estado do Pará. Sistemas de Produção - 3, dez. 2006. Disponível em: <http://sistemasdeproducao.cnptia.embrapa.br/ FontesHTML/BovinoCorte/BovinoCortePara/paginas/ manejo.html.>. Acesso em: 3 jan. 2010.

LAMPRECHT, H. Ensayo sobre la estrutura florística de laparte sur-oriental del bosque universitário: el Caimital, Estado Barinas. R. Flor. Venezuelana, v. 7, n. 10/11, p. 77-119, 1964.

LARA, J. F. R.; MACEDO, J. F.; BRANDÃO, M. Plantas daninhas em pastagens de várzeas no Estado de Minas Gerais. Planta Daninha, v. 21, n. 1, p. 11-20, 2003.
LORENZI, H. Plantas daninhas do Brasil: terrestres, aquáticas, parasitas e tóxicas. 3.ed. Nova Odessa: Plantarum, 2000. 608 p.

MACIEL, C. D. G. et al. Composição florística da comunidade infestante em gramados de Paspalum notatum no município de Assis, SP. Planta Daninha, v. 26, n. 1, p. 57-64, 2008.

MASCARENHAS, M. H. T. et al. Flora infestante em pastagem degradada sob recuperação, pelo sistema de integração lavoura-pecuária, em região de cerrado. R. Bras. Milho Sorgo, v. 8, n. 1, p. 41-55, 2009.

ORYOKOT, J. O. E.; MURPHY, S. D.; SWANTON, C. J. Effect of tillage and corn on pigweed (Amaranthus spp.) seedling emergence and density. Weed Sci., v. 45, n. 1, p. 120-126, 1997.

POTT, A.; POTT, V. J. Plantas do pantanal. Corumbá: Embrapa-CPAP/Brasília: Embrapa, 1994. 320 p.

SILVA, A. M. A.; COELHO, I. D.; MEDEIROS, P. R. Levantamento florístico das plantas daninhas em um parque público de Campina Grande, Paraíba, Brasil. R. Biotemas, v. 21, n. 4, p. 7-14, 2008.

SORENSEN, T. A method of stablishing groups of equal amplitude in plant society based on similarity of species content. In: ODUM, E.P. (Ed.). Ecologia. 3.ed. México: Interamericana, 1972. $640 \mathrm{p}$.

SVICERO, E.F.; BARROS, J.C.; NETO, A.L. Manejo de plantas daninhas em pastagens. 2008. Disponível em: <http:// www.planoconsultoria.com.br/site/circular14.doc.>. Acesso em: 30 set. 2009.

TUFFI SANTOS, L. D. et al. Levantamento fitossociológico em pastagens degradadas sob condições de várzea.

Planta Daninha, v. 22, n. 3, p. 343-349, 2004. 